\title{
Overexpression of PRDM13 inhibits glioma cells via Rho and GTP enzyme activation protein
}

\author{
LINNA ZHANG ${ }^{1}$, HUIMEI CAO $^{1}$, TAO HE $^{2}$, JIJUAN YANG $^{1}$, HONG TAO $^{1}$, YIN WANG $^{3}$ and QIKUAN HU $^{1,3}$ \\ ${ }^{1}$ Department of Physiology, Ningxia Medical University, Yinchuan, Ningxia 750004; \\ ${ }^{2}$ Department of Spinal Surgery, Dongfeng General Hospital of Chinese Medicine Affiliated to \\ Hubei Medical University, Shiyan, Hubei 442000; ${ }^{3}$ Ningxia Key Laboratory of Cerebrocranial Diseases, \\ Basic Medical School of Ningxia Medical University, Yinchuan, Ningxia 750004, P.R. China
}

Received November 20, 2017; Accepted May 10, 2018

DOI: $10.3892 /$ ijmm.2018.3679

\begin{abstract}
PR (PRDI-BFI and RIZ) domain containing (PRDM) proteins have been shown to be important in several types of human cancer. PRDM13, a member of the PRDM family, contains transcriptional regulators involved in modulating several cellular processes. However, the function of PRDM13 in glioma remains to be elucidated. The purpose of the present study was to evaluate the expression and effect of PRDM13 on glioma cells. It was found that the expression of PRDM13 was reduced in glioma cells, and the overexpression of PRDM13 significantly decreased the proliferation, migration and invasion of U87 glioma cells. Through validation of RNA-sequencing analysis, genes regulating cell proliferation and migration were classified from Gene Ontology sources. In addition, PRDM13 was shown to be associated with Rho protein and GTP enzyme activation protein. The overexpression of PRDM13 upregulated deleted in liver cancer 1 (DLC1) to inhibit the proliferation and invasion of U87 cells. In conclusion, PRDM13 decreased the proliferation and invasion of U87 cells, and may be of potential value for glioma therapy.
\end{abstract}

\section{Introduction}

The PRDI-BFI and RIZ homology domain containing (PRDM) family contains 17 orthologs in primates, and 16 orthologs in rodents, birds and amphibians, the regulation of which has been associated with cancer.

PRDM2 was the first tumor suppressor identified within the PRDMs whose expression causes apoptosis and/or cell cycle arrest in cancer cell lines, and suppresses tumor growth

Correspondence to: Professor Qikuan Hu, Ningxia Key Laboratory of Cerebrocranial Diseases, Basic Medical School of Ningxia Medical University, 1160 Shengli Street, Yinchuan, Ningxia 750004, P.R. China

E-mail: huqikuan@163.com

Key words: glioma, PRDI-BFI and RIZ homology domain containing 13, proliferation, invasion, U87 cell line in vivo $(1,2)$. PRDM1 was identified as a tumor suppressor of diffuse large B-cell lymphoma in two mouse models of PRDM1 deficiency in the B cell compartment (3). PRDM3 and PRDM16 have been identified as different isoforms associated with leukemia $(4,5)$, and PRDM5 is a potential tumor suppressor involved in cell adhesion and extracellular matrix formation along with several genes in the Wnt pathway (6-8). Therefore, several members of the PRDM family can suppress tumors.

Following Basic Local Alignment Search Tool searches of the National Center for Biology Information database, the present study focused on one of the PRDMs, PRDM13, which is expressed in the nervous system. PRDM13 encodes a protein containing an N-terminal PR domain and four zinc finger repeats, which mediate sequence-specific DNA binding and protein-protein interactions. However, to the best of our knowledge, neither the expression nor the role of PRDM13 in glioma has been reported. Glioma is a common central nervous system tumor, which is frequently life threatening, despite optimized treatment including neurosurgery, radiotherapy and chemotherapy. Therefore, it is necessary to understand the molecular mechanisms underlying the proliferation and migration of gliomas. In the present study, it was identified that the expression of PRDM13 was low in U87 cells, therefore, it was hypothesized that the upregulation of PRDM13 may be important for glioma development. Subsequently, the effects of PRDM13 on cell proliferation, migration and invasion were investigated in the U87 human glioma cell line. The results demonstrated that PRDM13 may upregulate anti-oncogenes to inhibit glioma progression. These findings suggested that the overexpression of PRDM13 may be a useful molecular target for treating human glioma.

\section{Materials and methods}

Cell lines and cell culture. The U87 cell line was purchased from the Cell Bank of the Chinese Academy of Sciences (Shanghai, China; http://www.biovector.net/search?wd=U87). The cells were routinely cultured in Dulbecco's modified Eagle's medium (Gibco; Thermo Fisher Scientific, Inc., Waltham, MA, USA); all media contained $10 \%$ fetal calf serum (Biochrom, Ltd., Cambridge, UK) and $1 \%$ penicillin/streptomycin (Invitrogen; 
Thermo Fisher Scientific, Inc.), and the cells were cultured at $37^{\circ} \mathrm{C}$ in an atmosphere containing $5 \% \mathrm{CO}_{2}$.

Lentivirus construction and transfection. The PRDM13 lentivirus construct was designed by Shanghai GeneChem Co., Ltd. (Shanghai, China) and a GFP lentivirus was used as the negative control (NC). PRDM13 was inserted into the Ubi-MCS-3FLAG-SV40-EGFP-IRES-puromycin lentiviral vector. The U87 cells were plated in a six-well plate and the cells were transfected with PRDM13 lentivirus and NC lentivirus (LV control) at a multiplicity of infection of 10 with polybrene (Sigma-Aldrich; Merck Millipore, Darmstadt, Germany). Non-transfected cells were used as controls. The cells were cultured in a $5 \% \mathrm{CO}_{2}$ incubator at $37^{\circ} \mathrm{C}$ for a further 7 days. The expression of GFP was observed by fluorescence microscopy.

Reverse transcription-quantitative polymerase chain reaction (RT-qPCR) analysis. Total RNA was extracted using the RNA spin Mini RNA isolation kit (Axygen Scientific, Inc., Union City, CA, USA). cDNA was synthesized with the Promega Reverse Transcriptase system (Promega Corporation, Madison, WI, USA). The PCR amplification was performed using Taq Master mix (TransGen Biotech Co., Ltd., Beijing, China). Thirty-one primer pairs of different genes were designed and tested. The PCR cycling conditions were as follows: $94^{\circ} \mathrm{C}$ for $30 \mathrm{sec}$, followed by 40 repeats of $94^{\circ} \mathrm{C}$ for $5 \mathrm{sec}$ and $60^{\circ} \mathrm{C}$ for $30 \mathrm{sec}$. The relative expression levels of the genes of interest were normalized to the levels of GAPDH. The results were quantified as target gene/GAPDH using the $2^{-\Delta \Delta \mathrm{Cq}}$ method (9). The primers used are shown in Table I. All measurements were performed in triplicate.

Western blot analysis. The cells were washed twice with cold PBS, and then lysed in cold lysis buffer. The mix solution was centrifuged at $29,040 \mathrm{~g}$ for $50 \mathrm{~min}$ at $4^{\circ} \mathrm{C}$. Following collection of the supernatant, the protein concentration was determined with the BCA method (Nanjing KeyGen Biotech Co., Ltd., Nanjing, China), according to the manufacturer's protocol. A total of $50 \mu \mathrm{g}$ of protein was loaded and separated by $8-10 \%$ SDS-PAGE, according to molecular weight, and then transferred onto PVDF membranes. Subsequently, the membranes were incubated with antibodies at $4^{\circ} \mathrm{C}$ overnight. The antibodies used were as follows: PRDM13 (1:1,000; cat. no. ab40542), Flag (1:1,000; cat. no. ab192404), and deleted in liver cancer 1 (DLC1; 1:1,000; cat. no. ab1220) from Abcam (Cambridge, MA, USA), Rho GTPase activating protein 30 (ARHGAP30; 1:500; cat. no. AP4996b; Abgent Biotech Co., Ltd., Wuhan, China), A disintegrin and metalloproteinase with thrombospondin motifs 12 (ADAMTS12; 1:500; cat. no. 24934-1-AP; ProteinTech Group, Inc., Wuhan, China), inhibitor of cyclin-dependent kinase (CDK) interacting with cyclin A1 (INCA1; 1:500; cat. no. bsm-7899R; BIOSS, Beijing, China), and GAPDH (1:6,000; cat. no. 60004-1-Ig; ProteinTech Group, Inc.). The membranes were washed and incubated with the secondary antibodies $(1: 10,000)$ at room temperature for $90 \mathrm{~min}$, comprising goat anti-rabbit IgG (cat. no. bsm-0295G) and goat anti-mouse IgG (cat. no. bsm-0296G) from BIOSS. All antibodies were diluted in 5\% skim milk (BD Difco, cat. no. 2332100, SBJBio, Nanjing, China). Finally, the membranes were developed and quantified using Odyssey CLx Infrared Imaging system software, Image Studio Version 4.0 (LI-COR Biosciences, Lincoln, NE, USA).

Cell proliferation assay. Following transfection with lentiviruses expressing PRDM13 or the scramble control using polybrene, the U87 cells were cultured for $24 \mathrm{~h}$. The cells were collected and seeded in 96-well plates at a density of 2,000 cells/well. Cell proliferation rates were determined once per day for 5 days using a Cell Counting Kit-8 kit (TransGen Biotech Co., Ltd.). The samples were assayed in triplicate and OD values were detected at $450 \mathrm{~nm}$.

Flow cytometric analysis of cell cycle. The U87 cells were transfected with lentiviruses expressing PRDM13 or the scramble control. Following incubation for $72 \mathrm{~h}$, the cells were washed twice with PBS solution, trypsinized and fixed with pre-cooled $70 \%$ alcohol at $4^{\circ} \mathrm{C}$ overnight. The cells were washed with PBS and stained with propidium iodide (Cell Cycle Detection kit; KeyGen Biotech Co., Ltd.) at $4^{\circ} \mathrm{C}$ for $30 \mathrm{~min}$. Flow cytometry was performed using a FACSCalibur (BD Biosciences, Franklin Lakes, NJ, USA).

Wound-healing assay. The U87 cells were seeded in 60-mm cell culture plates and incubated at $37^{\circ} \mathrm{C}$ overnight in DMEM with $10 \%$ FBS. Upon reaching $90 \%$ confluence, the cells were cultured in serum-free medium for $24 \mathrm{~h}$. A $200-\mu 1$ sterile pipette tip was used to create a ' + ' wound. The cells were washed twice with PBS to remove cell debris and grown in FBS-free medium. Images of the wounds were captured under a microscope and repeated three times in duplicate.

Transwell invasion assay. Cell invasion was measured using BD BioCoat ${ }^{\mathrm{TM}}$ Matrigel $^{\mathrm{TM}}$ Invasion Chambers (BD Biosciences). Transwell chambers were coated with Matrigel at a final concentration of $1 \mathrm{mg} / \mathrm{ml}$. A total of $1 \times 10^{4}$ cells $/ \mathrm{ml}$ were seeded into the upper chamber with $100 \mu \mathrm{l}$ serum-free DMEM, and $500 \mu 1$ DMEM with $10 \%$ FBS medium was added into the lower chamber. The cells were incubated in a humidified environment with $5 \% \mathrm{CO}_{2}$ at $37^{\circ} \mathrm{C}$ for $24 \mathrm{~h}$. The cells on the top surface of the membrane were removed with a cotton swab, and the migratory cells on the bottom surface of the membrane were fixed in $90 \%$ alcohol for $30 \mathrm{~min}$ at room temperature. The chambers were washed with PBS and stained with $5 \%$ crystal violet for $30 \mathrm{~min}$. Migratory cells in the bottom surface of the membrane were counted by light microscopy. A total of six high-power microscopic fields (magnification, x200) were randomly selected and images captured, and the cells were counted.

Transcriptome sequencing. The total RNA of cells with lentiviruses expressing either PRDM13 or the scramble control was extracted using TRIzol reagent. The integrity of total RNA was examined using a NanoDrop 2000 spectrophotometer (Thermo Fisher Scientific, Inc.). Transcriptome sequencing was performed by Vazyme Biotech Co., Ltd. (Nanjing, China). Significant differentially expressed genes and the gene ontology (GO) function enrichment were obtained between two groups. 
Table I. Primer sequences for reverse transcription-quantitative polymerase chain reaction analysis

\begin{tabular}{|c|c|c|}
\hline Gene & Sense $\left(5^{\prime}-3^{\prime}\right)$ & Antisense (5'-3') \\
\hline RORA & AACGGGCTGACGGAACTTCA & GGGAAGGCTGTATGTCCAGGT \\
\hline EGR1 & TTCCCTGAGCCACAAAGCCA & GGAACCTGGAAGCCACCCTT \\
\hline WNT7B & GTGGACGCTCGGGAGATCAA & TCCTGCCGGCCTCATTGTTA \\
\hline BHLHE41 & GAGAACGACACGGACACCGA & GGAGGCTCCTGCTTGATGGT \\
\hline ROS1 & СТCCCAGCTGCACCСТCTG & TGAGAGCCAAACAGCACCGA \\
\hline PLEKHG5 & AGAGCTCTGCCTGGCTGTTC & AGCAGGGTGGTCCTGATTCG \\
\hline SNAI2 & GCACTGCGATGCCCAGTCTA & TGCCGCAGATCTTGCAAACA \\
\hline SEMA5A & СCTTGCCTTGGCCCATCTCT & GAGCAAGAGCGGGTCCTCAT \\
\hline TWIST1 & CCCTCGGACAAGCTGAGCAA & TCGCTCTGGAGGACCTGGTA \\
\hline ASAP3 & GTGAAGAGCAGGCCTGGGAA & GAGTGACTGCATGCGCGAAA \\
\hline ATOH8 & GCTGTCCAAACTGGCCATCC & TTGCTGTGGTCGGCACTGTA \\
\hline GADD45B & GAGGCCCGAGACCTGCATT & CACCAAGCCGTGGCTCTTC \\
\hline PIM1 & GTGCCCATGGAAGTGGTCCT & AACTGTCGGGCCTCTCGAAC \\
\hline ARHGAP30 & TGGGATGGGCTACCTGGAG & TGCCTCATCCAGAGACAGGT \\
\hline INCA1 & AAGTGTTCCAGGGTGGTCAG & GGTGGGCCTTTGATTAAGGT \\
\hline GRPR & GACAGTGCTGGTGTTTGTGG & TGACAAAGTGGAGCATGGAG \\
\hline IL24 & GCCAGCAAGCTCAGGATAAC & ATAGCAGAAACCGCCTGTGT \\
\hline ADAMTS12 & GTGGGAAACAGTGGCAAGAT & CAAGGATTGGGAAGTGAGGA \\
\hline DIRAS3 & GCCTTCATGGAGATTTCAGC & GCATCTGGGATTTCTTCTCG \\
\hline SLIT2 & GATGTGGCCATTCAGGACTT & TTGTTGCTACATCGGACGAC \\
\hline TFPI2 & CGGATTGAGAACAGGTTTCC & ATTGTCATTCCCTCCACAGC \\
\hline CTNNBIP1 & ATGGGATCAAACCTGACAGC & ATCACCACGTCCTCTGCAC \\
\hline EPHB 1 & ССТСССТАATGTCCCAGGAT & CCTCAGACCAGAAGGCTGAC \\
\hline CXCL14 & CTACAGCGACGTGAAGAAGC & CGCTCTTGGTGGTGATGATA \\
\hline COL4A6 & CTGGCTTTCTTGGCATCAAT & CCAACAGCTCCTTGAGTTCC \\
\hline ADAMTS6 & AAGTCTGCCCTTCAACAACG & ATGTGTCATTCAGCCACCAA \\
\hline DLC1 & CCAGCAACTTGGCAGGCAAT & GCTTCAGGCTCTCCATCCGT \\
\hline ITGBL1 & GTGGTCGCTGTGTGTTTGTGAG & CGAGCACAATATGCCATCTG \\
\hline WNT10B & CATCCTCAAGCGCGGTTTCC & CTCAGCCGATCCTGCTCACC \\
\hline SOX7 & ATGGTTTGGGCCAAGGACGA & CAGCGCCTTCCACGACTTTC \\
\hline GAPDH & AGAAGGCTGGGGCTCATTTG & AGGGGCCATCCACAGTCTTC \\
\hline
\end{tabular}

Validation of RNA-sequencing (RNA-Seq) analysis by $R T-q P C R$ and western blot analyses. According to the transcriptome sequencing results, genes of interest were selected relative to the proliferation and migration function with the following criteria: $\mathrm{P}<0.05$ and absolute fold change $>2$. RT-qPCR and western blot analyses were performed to validate the RNA-Seq analysis. The primers used are listed in Table I, and the methods for RT-qPCR and western blot analyses were as described above.

Chromatin immunoprecipitation (ChIP) assay. The ChIP assay was performed using the Pierce ${ }^{\mathrm{TM}}$ Agarose ChIP kit (cat. no. 26156; Thermo Fisher Scientific, Inc.), according to the manufacturer's protocol. Immunoprecipitated DNA enrichment was normalized relative to the input. The enrichment of each target sequence was determined by RT-qPCR analysis. Flag antibody (1:1,000; cat. no. ab192404, Abcam) was used to perform ChIP. The primers used were as follows: DLC1 ChIP, forward 1 5'-CTCTCCGGAGGTCCTTTAGC-3'; DLC1
ChIP, reverse 1 5'-CCAGCCGTCTGTCTCTAGTGT-3'; DLC1 ChIP, forward 2 5'-CTTTGAAAACGCGAGGTCAC-3'; and DLC1 ChIP, reverse 2 5'-TGCAACCCAATGACTCACCT-3'. All samples were assessed in triplicate.

Statistical analysis. All data are expressed as the mean \pm standard deviation. Statistical analyses were performed using SPSS 17.0 software (SPSS, Inc., Chicago, IL, USA). Comparisons between the NC group and the PRDM13 group were made using Student's t-test. $\mathrm{P}<0.05$ was considered to indicate a statistically significant difference.

\section{Results}

Efficient transfection of Lenti-PRDM13 into the U87 cell line. The lower expression of PRDM13 in U87 human glioma cells suggested that PRDM13 may be involved in glioma development. To examine the role of PRDM13 in gliomas, the Lenti-PRDM13 and Lenti-Con (NC) lentiviruses were 
transfected into U87 cells. The successful transfection of Lenti-PRDM13 or Lenti-Con into the U87 cells was confirmed by microscopic green fluorescence detection. As shown in Fig. 1, the transfection efficiency was $>80 \%$ by 3 days post-infection with lentivirus. The RT-qPCR data demonstrated that the mRNA expression level of PRDM13 was significantly increased in the Lenti-PRDM13-infected cells $(\mathrm{P}<0.01$; Fig. 2A). In addition, the protein expression level of Flag-PRDM13, detected by western blot analysis, was markedly increased in the Lenti-PRDM13-infected cells, compared with that in the NC cells ( $\mathrm{P}<0.01$; Fig. 2B). These data indicated that the PRDM13 gene was effectively overexpressed at the mRNA and protein levels.

Effects of the overexpression of PRDM13 on U87 cells. Tumor cell proliferation is a vital characteristic of tumors. Therefore, it was necessary to examine the impact of the overexpression of PRDM13 on cell proliferation in the U87 cell line. It was demonstrated that cell proliferation was decreased from the third day in the cells infected with Lenti-PRDM13, compared with that in the NC cells (Fig. 3A). The role of PRDM13 in the cell cycle of the U87 cells was analyzed by flow cytometry (Fig. 3B). It was demonstrated that the cell percentage in the $\mathrm{G}_{0} / \mathrm{G}_{1}$ phase was increased from $66.66 \%$ in the $\mathrm{NC}$ group to $77.34 \%$ in the Lenti-PRDM13-infected cells, and the cell percentage in the $\mathrm{S}$ phase was decreased to $15.81 \%$ from $25.83 \%(\mathrm{P}<0.05)$. These results indicated that PRDM13 inhibited DNA duplication in the U87 human glioma cells.

The results of the wound-healing assay demonstrated that the migration of U87 cells was lower in the PRDM13 overexpression group than in the NC group (Fig. 4A). The results from the Transwell invasion assay demonstrated that the overexpression of PRDM13 suppressed the invasion of U87 cells (Fig. 4B). The average invasive cell number was 28 in the PRDM13 overexpression group, and 75 in the NC group $(\mathrm{P}<0.01$; Fig. 4B). These data showed that the overexpression of PRDM13 may suppress the migration and invasion of U87 cells.

Analysis of transcriptome sequencing data. To further investigate the mechanisms underlying the effect of the overexpression of PRDM13 in U87 glioma cells, transcriptome sequencing data were analyzed in order to reveal the molecular mechanisms of U87 cells infected with Lenti-PRDM13 or Lenti-control. The volcano plot revealed 760 differentially expressed genes between the two groups, including 222 upregulated genes and 538 downregulated genes $(\mathrm{P}<0.05$; Fig. 5A). GO analysis revealed three differentially expressed gene functional enrichments: Molecular function, cellular component and biological process. The genes were primarily involved in 'receptor binding', 'molecular function regulator', 'extracellular region part', 'cell periphery', 'system development', 'single-multicellular organism process', 'regulation of multicellular organismal process', 'anatomical structure development' and 'regulation of cell migration' (FDR<0.05; Fig. 5B).

Significant differential expression in genes between the two groups was analyzed. Following consultation of the relevant literature, it was identified that a number of these genes were involved in tumorigenesis, proliferation and migration,

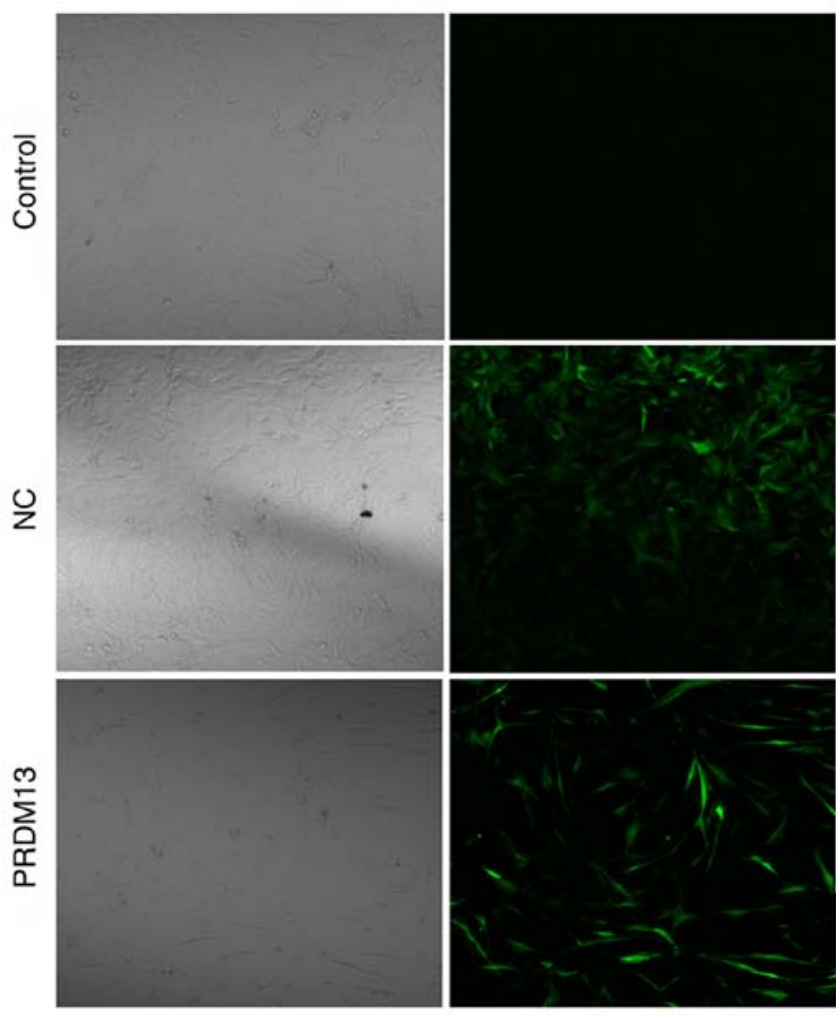

Figure 1. Lentivirus-mediated overexpression of the PRDM13 gene in U87 glioma cells. The U87 cells were transfected with Lenti-Con or Lenti-PRDM13. In total, $>80 \%$ of U87 cells expressed GFP. Light (left) and fluorescence (right) micrographs of human glioma U87 cells (magnification, $\mathrm{x} 100$ ) in non-transfected control, NC and PRDM13 overexpresssion groups. PRDM13, PRDI-BFI and RIZ homology domain containing 13; NC, negative control.

including SRY-Box 7 (SOX7), tumor necrosis factor receptor superfamily member 8 (TNFRSF8), RAR-related orphan receptor $\alpha$ (RORA), early growth response 1 (EGR1), basic helix-loop-helix family, member e41 (BHLHE41), Snail family transcriptional repressor 2 (SNAI2), ArfGAP with SH3 domain, ankyrin repeat and PH domain 3 (ASAP3), Growth arrest and DNA-damage-inducible $\beta$ (GADD45B), PIM1, ADAMTS12, integrin $\beta$-like 1 (ITGBL1), DLC1 and ADAMTS6. These genes have been demonstrated to have a promoting or inhibitory effect on tumors. A number of known inflammatory and immune-associated genes, including interleukin (IL)24, chemokine (C-X-C motif) ligand 14 and interleukin 1 receptor like 1 , were also identified. The results of the analyses of these genes is likely to facilitate investigations into the associated mechanisms.

Anti-oncogenes are involved in the PRDM13-mediated progression of glioma cells. According to the results of transcriptome sequencing data, genes associated with the regulation of cell proliferation and migration were identified from GO sources. A total of 15 upregulated genes and 15 downregulated genes were validated by RT-qPCR analysis. All genes coincided with the results of the transcriptome sequencing data (Fig. 6A). Of note, the anti-oncogenes DLC1, ARHGAP30, INCA1 and ADAMTS12 were all upregulated at the protein level (Fig. 6B). The ChIP assay demonstrated that PRDM13 may bind to the DLC1 promoter to regulate 

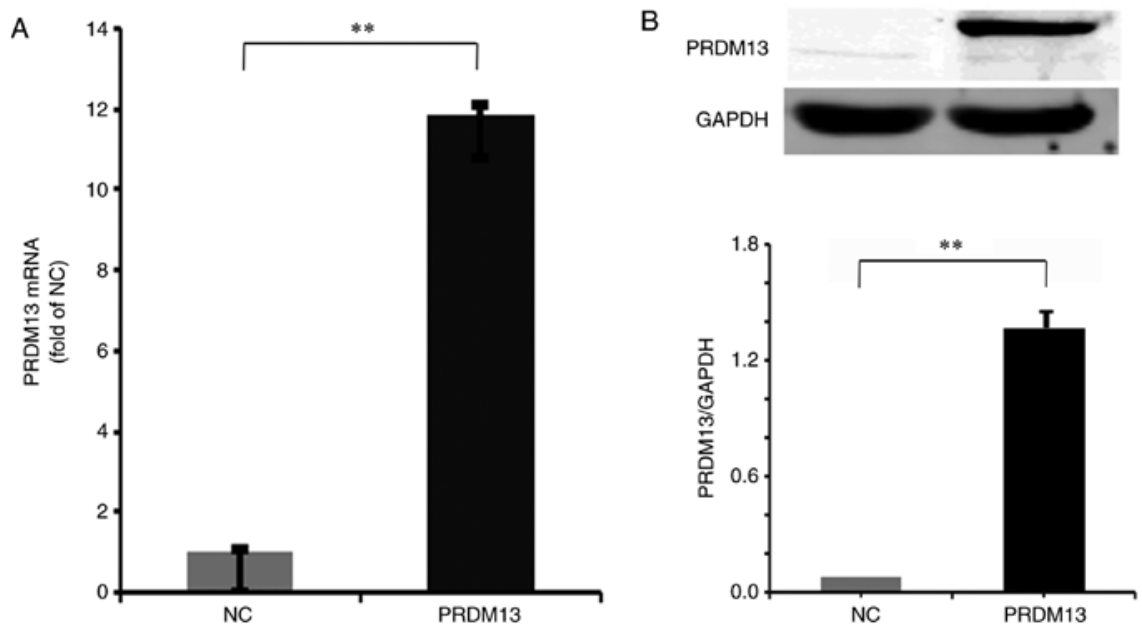

Figure 2. Gene expression of PRDM13 in selected human glioma cell lines. mRNA and protein overexpression levels of PRDM13 were detected by RT-qPCR and western blot analyses, respectively, in U87 cells following lentiviral transfection. (A) U87 cells were infected with a lentivirus expressing either PRDM13 or Con and cultured for 7 days. Total RNA was extracted for RT-qPCR analysis to quantify the overexpression of PRDM13 at the mRNA level. The mRNA level of PRDM13 was normalized to that of GAPDH. (B) Overexpression of PRDM13 at the protein level was analyzed by western blot analysis in U87 cells cultured for 7 days. The total proteins were isolated, and PRDM13 protein was detected using PRDM13 and Flag antibodies. GAPDH was used as an internal control. ${ }^{* *} \mathrm{P}<0.01$. NC, negative control; PRDM13, PRDI-BFI and RIZ homology domain containing 13; RT-qPCR, reverse transcription-quantitative polymerase chain reaction.
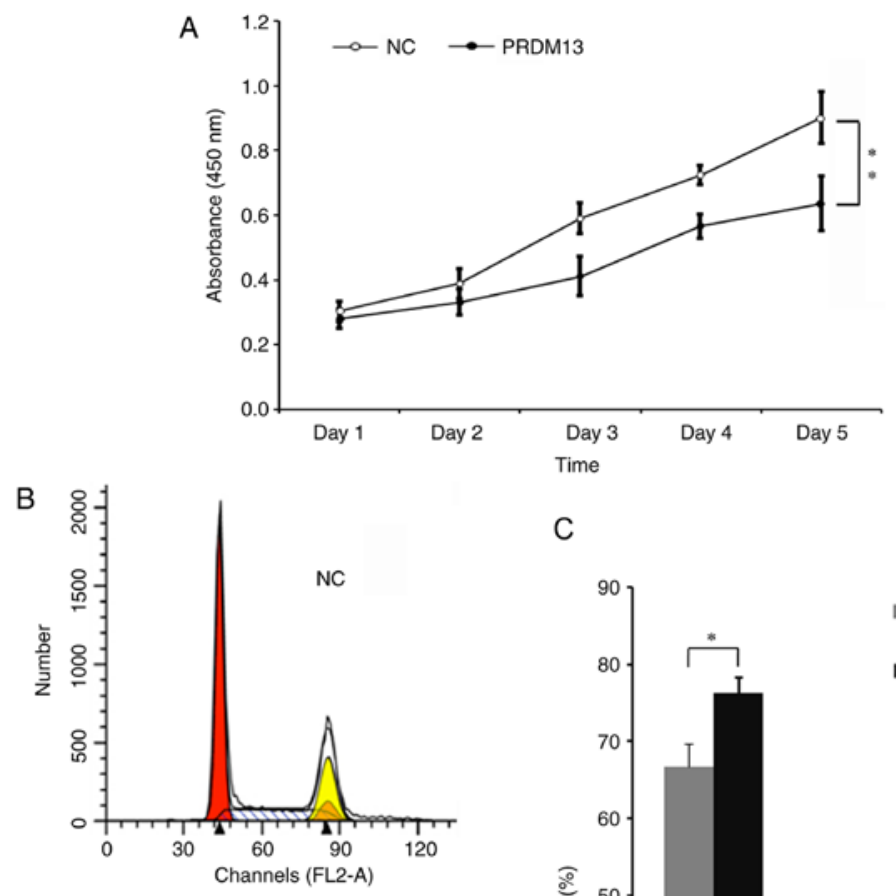

C
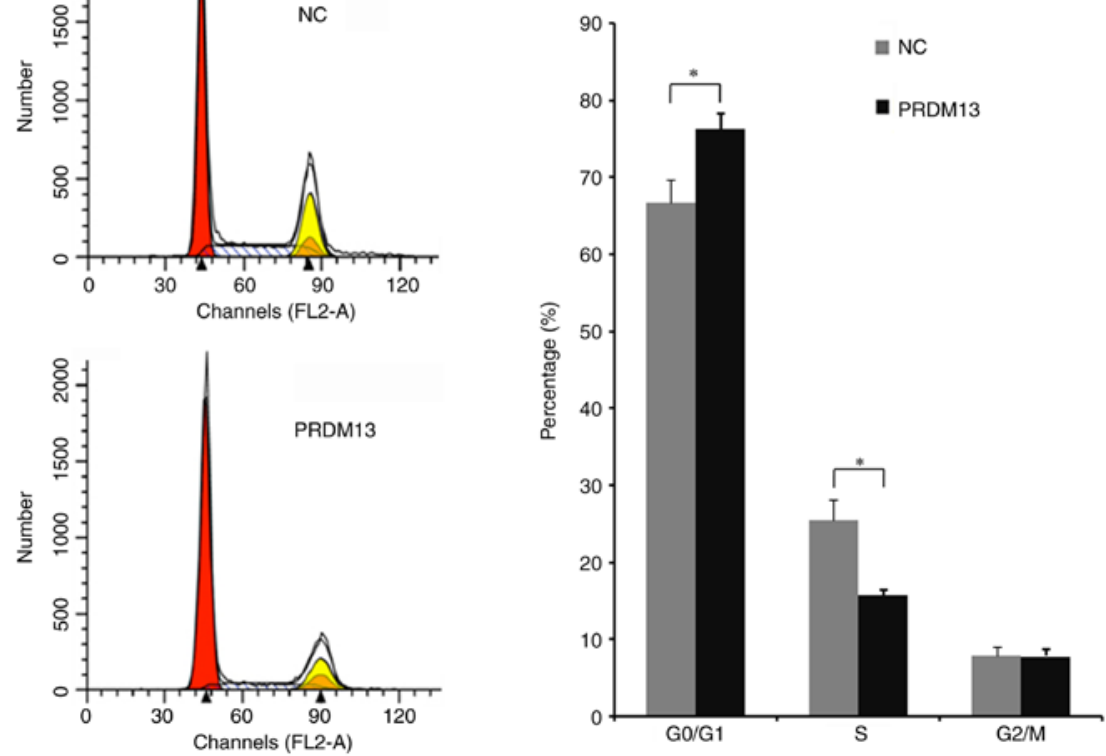

Figure 3. PRDM13 influences tumor cell proliferation and cell cycle arrest. (A) Overexpression of PRDM13 using a lentivirus in U87 cells decreased the cell proliferation rate, according to the Cell Counting Kit-8 assay. Cells were infected with Lenti-PRDM13 or Lenti-Con and cultured for 5 days. (B) Images of cell cycle distribution in U87 cells infected with Lenti-PRDM13 or Lenti-Con by flow cytometry and propidium iodide staining. (C) Percentages of cells in the different phases $\left(\mathrm{G}_{0} / \mathrm{G}_{1}, \mathrm{~S}\right.$ and $\mathrm{G}_{2} / \mathrm{M}$ phase) of the cell cycle in U87 cells infected with Lenti-PRDM13 or Lenti-Con. ${ }^{*} \mathrm{P}<0.05$, ${ }^{* * *} \mathrm{P}<0.01$. PRDM13, PRDI-BFI and RIZ homology domain containing 13; NC, negative control. 

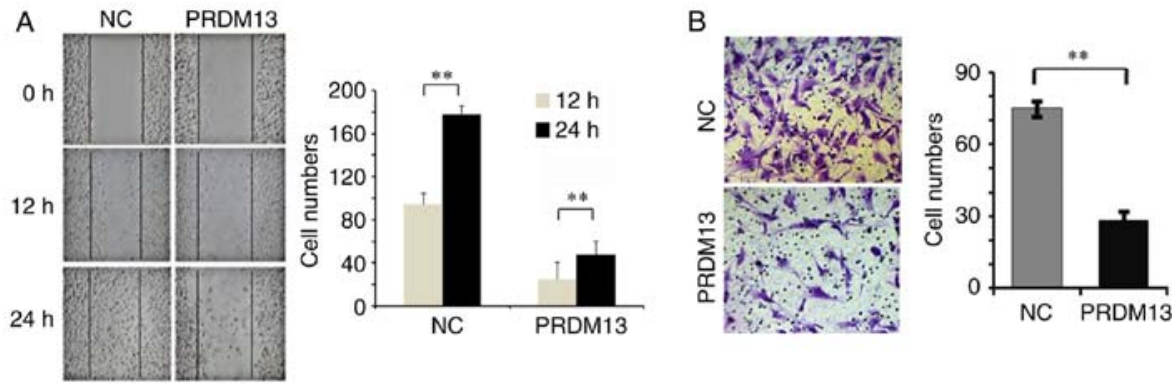

Figure 4. Overexpression of PRDM13 reduces migration and invasion of U87 cells. (A) Migratory ability of U87 cells was examined using a wound-healing assay at 12 and $24 \mathrm{~h}$. (B) Invasive ability of U87 cells was examined using a Transwell assay. Lenti-PRDM13-infected cells exhibited limited invasion (left) Randomly selected microscopic fields were calculated (right). Magnification, $x 200 .{ }^{* *} \mathrm{P}<0.01$. PRDM13, PRDI-BFI and RIZ homology domain containing 13; NC, negative control.

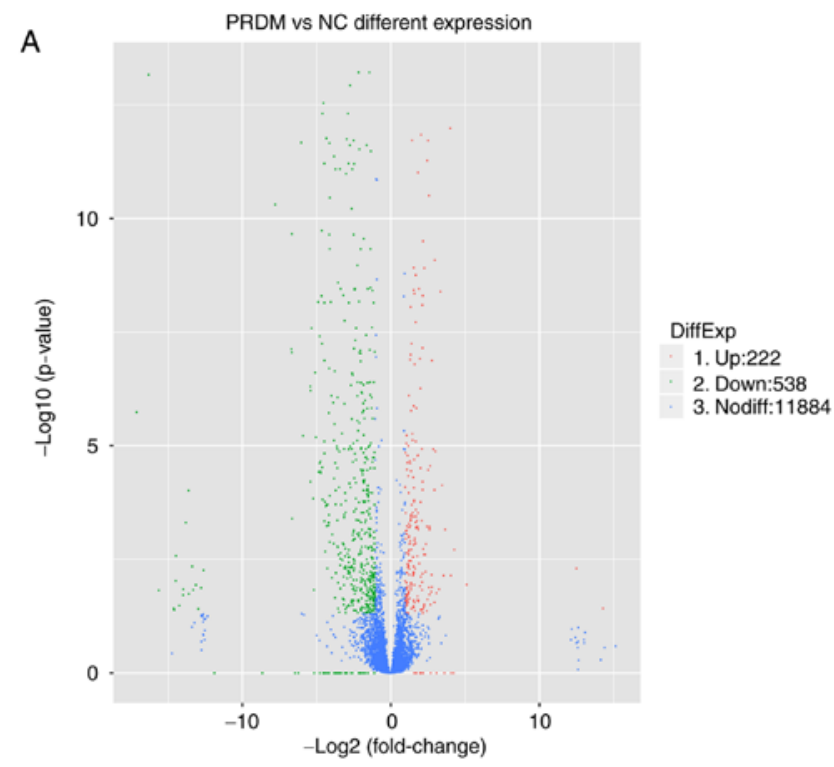

B

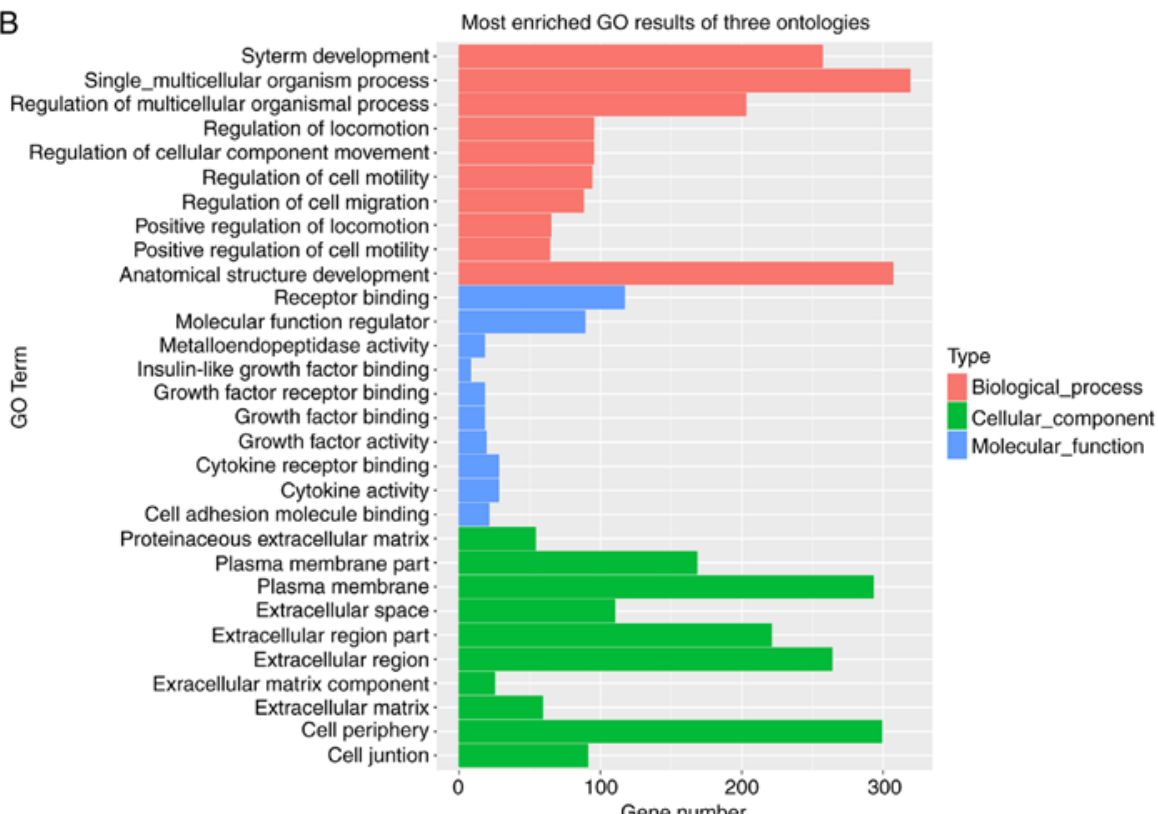

Figure 5. Analysis of gene expression changes in U87 cells with overexpression of PRDM13 by transcriptome sequencing. (A) Volcano plot shows differentially expressed genes in the U87 human malignant glioma cell line infected with a lentivirus expressing either PRDM13 or Lenti-con under the criteria of $\mid \log 2$ Ratiol $\geq 1$ and $P \leq 0.05$. The genes are denoted by different colored dots. The fold change is shown at the bottom in the image (red represents upregulated genes, green represents downregulated genes, and blue represents no difference). (B) Functional enrichment was analyzed via GO. The x-axis represents the number of significantly different genes and the y-axis represents the GO term. Different colors represent different categories of genes (red represents biological process, blue represents cellular component and green represents molecular function). PRDM13, PRDI-BFI and RIZ homology domain containing 13; NC, negative control; GO, gene ontology; DiffExp, differential expression; up, upregulated; down, downregulated; nodiff, no difference. 

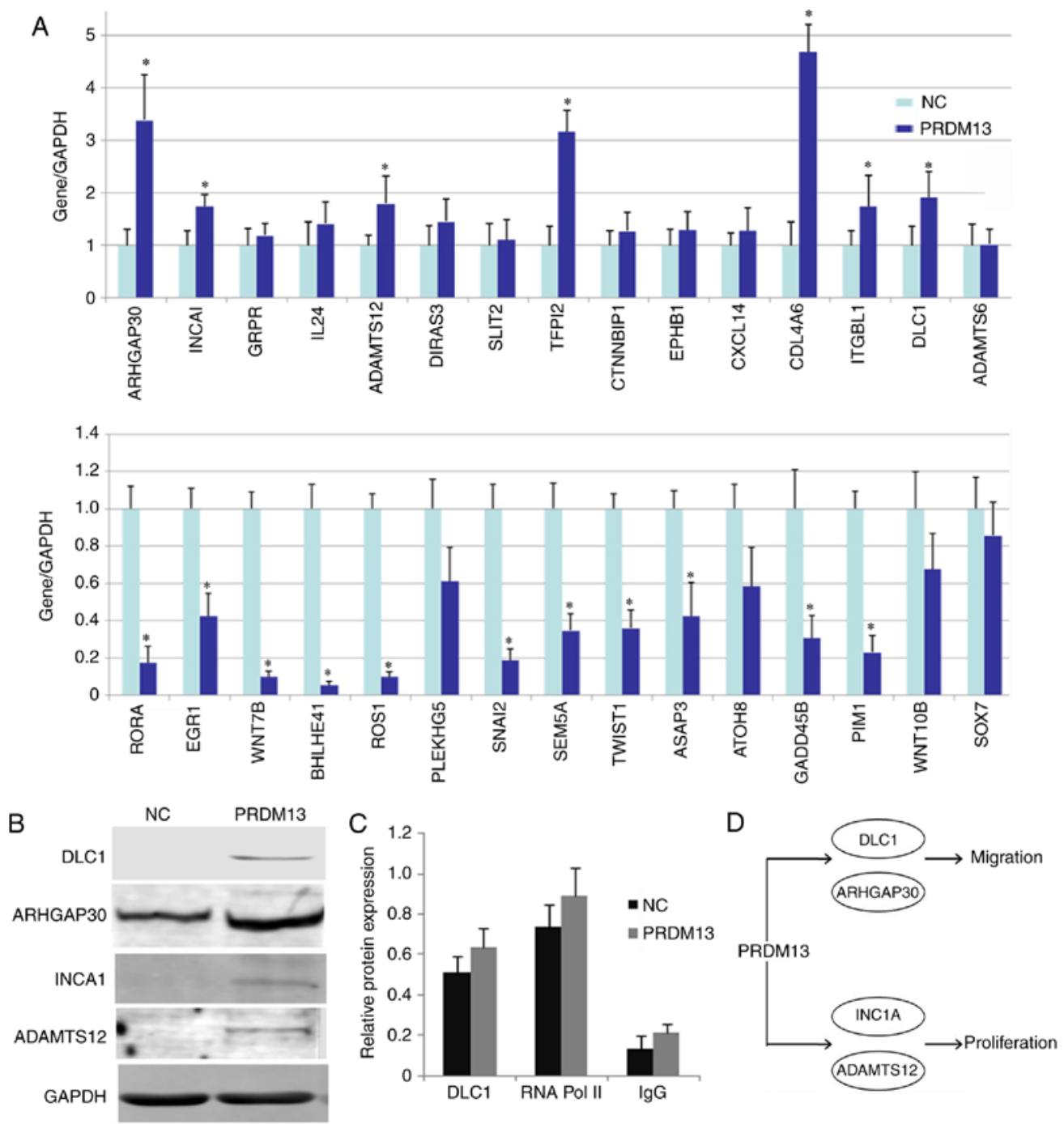

Figure 6. Proliferation and migration-associated genes involved in U87 cells infected with Lenti-PRDM13 and Lenti-Con. (A) Total RNA was extracted and reverse transcription-quantitative polymerase chain reaction analysis was performed to quantify the expression of different proliferation- and migration-associated genes at the mRNA level. The mRNA level was normalized to that of GAPDH. "P<0.05 (B) Protein expression levels of DLC1, ARHGAP30, INCA1 and ADAMTS12 were analyzed by western blot analysis in U87 cells. GAPDH was used as an internal control. (C) Chromatin immunoprecipitation assay in U87 cells exhibited a direct association between PRDM13 and the DLC1 promoter. (D) Diagram summarizing the conclusion that PRDM13 may upregulate DLC1 and ARHGAP30, which suppress U87 cell migration and upregulate INCA1 and ADAMTS12, causing a decrease in U87 cell proliferation. PRDM13, PRDI-BFI and RIZ homology domain containing 13; DLC1, deleted in liver cancer 1; ARHGAP30, Rho GTPase activating protein 30; ADAMTS12, A disintegrin and metalloproteinase with thrombospondin motifs 12; INCA1, inhibitor of cyclin-dependent kinase interacting with cyclin A1; $\mathrm{NC}$, negative control.

its expression (Fig. 6C). As summarized in the diagram in Fig. 6D, PRDM13 may upregulate DLC1 and ARHGAP30, suppressing U87 cell migration, upregulating INCA1 and ADAMTS12, and causing a decrease in U87 cell proliferation.

\section{Discussion}

The PRDM family members have been reported to correlate closely with cancer, and the majority are tumor suppressors. PRDM13 is a member of the PRDM family that contains a PR domain and a zinc finger motif (10). However, the biological function of PRDM13 in glioma has not been elucidated previously. Previous studies have demonstrated that PRDM13 is a transcriptional repressor, which regulates the excitatory/inhibitory balance (11). In the present study, lower expression levels of PRDM13 were identified in glioma cells and glioma tissues, therefore, the role of PRDM13 in glioma cells was examined. The results demonstrated that the overexpression of PRDM13 inhibited the proliferation, migration and invasion of U87 cells, and decreased the percentage of cells in the S phase of the cell cycle, suggesting that PRDM13 may influence DNA replication.

Dysregulation of the cell cycle is one of the important reasons behind the malignant transformation and uncontrolled proliferation of cells and, ultimately, the formation of malignant tumors. INCA and CDK inhibitor may inhibit the activity of CDK2 and cell proliferation. This inhibition depends on cell cycle protein interactions. Mitogen and oncogenic signaling may inhibit INCA1, which is caused by cell cycle arrest. Studies have identified that the expression of novel CDK inhibitor INCA1 was decreased in acute leukemia myeloid and lymphoid cells. This gene is important for cell 
survival, including genetic damage detection and repair (12). The expression of ADAMTS12 has been reported to be associated with a tumor cell anti-proliferation effect by modulating the extracellular signal-regulated kinase signaling pathway. It has also been identified as a novel antitumor protease. It simultaneously induces interstitial cells that may act as part of normal tissues in controlling cancer progression (13). In the absence of ADAMTS12, angiogenesis and tumor invasion in host tissues increase. El Hour et al (14) reported that ADAMTS12 exhibited anti-angiogenic properties and promoted host defenses against tumors. The expression of ADAMTS12 is associated with tumor histological type, tumor invasion and lymph node metastasis. If the expression of ADAMTS12 is low or missing in the extracellular matrix, the survival rate of the patient is relatively poor. ADASTM12 is important in tumor development; its expression may be a good prognostic indicator in colon cancer (15). The PRDM13-mediated inhibition of proliferation in glioma is associated with the above proteins.

Among tumor characteristics, invasion and growth are vital for glioma tumors (16). The results of the present study demonstrated that the overexpression of PRDM13 inhibited the migration and invasion of glioma cells. In order to obtain further insights into the mechanism of PRDM13-associated glioma progression, RNA-Seq analysis for U87 cells overexpressing PRDM13 was performed, and hundreds of genes exhibited significant differential expression. Among them, the gene expression levels of Rho protein and GTP enzyme activation protein were examined in U87 cells. The overexpression of PRDM13 in U87 cells induced upregulation of the expression of DLC1 and ARHGAP30. ChIP was performed to show that PRDM13 was involved with the transcription start site to upregulate the expression of DLC1. These results indicated that PRDM13 inhibited the migration of glioma cells through the upregulation of Rho family proteins.

The Rho family proteins function as important regulators in the organization of the actin cytoskeleton, and dysregulation of the Rho GTPase-associated signaling pathways has been reported in tumors (17). Low expression levels of ARHGAP30, a Rho GTPase-activating protein, have been reported to be associated with poor survival rates in patients with colorectal cancer (CRC) (18). ARHGAP30 is required for p53 acetylation and functional activation in $\mathrm{CRC}$, and the expression of ARHGAP30 is a potential prognostic marker for CRC (18). DLC1, which is associated with human HCC, is also termed ARHGAP7 and is a Rho GTPase-activating protein. DLC1 has been found to be downregulated in a number of cancer tissues and cells. It has been reported that DLC1 is a tumor suppressor gene in liver cancer in addition to other types of cancer $(19,20)$. The DLC-1 gene, deleted in liver cancer at chromosome 8p21-22, is altered primarily by genomic deletion or aberrant promoter methylation in a large number of types of human cancer, including breast, liver, colon and prostate cancer, and is known to have an inhibitory effect on breast and liver tumor cell growth (21). A feature of many advanced tumors is the activation of the Rho-GTPases RhoA and RhoC. These small GTPases, which are Ras-related proteins, may contribute to various parameters of abnormal cell growth, including viability, migration, invasion, proliferation and anchorage-independent growth (22-24). GTPase-activating protein expression is frequently downregulated or silenced in a variety of human malignancies. The observations in the present study revealed that the overexpression of PRDM13 is likely to be associated with the GTPase-activating proteins, inhibiting glioma cell migration. In the future, it may be worthwhile to investigate the mechanism underlying PRDM1-mediated Rho protein expression.

In conclusion, the present study demonstrated that PRDM13 was important in glioma cell malignant progression in vitro, as the overexpression of PRDM13 prevented proliferation, migration and invasion. Verhaak et al (25) reported the gene expression-based molecular classification of GBM into proneural, neural, classical, and mesenchymal subtypes. Aberrations and gene expression of epidermal growth factor receptor, neurofibromin 1, and platelet derived growth factor receptor $\alpha /$ isocitrate dehydrogenase 1 each define the different subtypes, Their findings were from brain tissues of patients. The findings of the present study are significant, however, the role of PRDM13 on glioma cells was discussed using the U87 cell line in vitro only. This was a limitation of the present study and future investigations into the detailed molecular mechanisms of the role of PRDM13 in glioma are required. These results provide the biological basis for a novel therapeutic approach in glioma.

\section{Acknowledgements}

Not applicable.

\section{Funding}

The present study was supported by grants from the National Natural Science Foundation of China (grant no. 301460300) and West China Top Class Discipline Project in Basic Medical Sciences, Ningxia Medical University (grant no. NXYLXK2017B07).

\section{Availability of data and materials}

The datasets used and/or analyzed during the current study are available from the corresponding author on reasonable request.

\section{Authors' contributions}

LZ, YW and QH designed the study. LZ, HC, TH, JY and HT performed the research. LZ, HC, TH and QH analyzed the data. LZ, YW and QH wrote and revised the manuscript. All authors read and approved the final manuscript.

\section{Ethics approval and consent to participate}

Not applicable.

\section{Consent for publication}

Not applicable.

\section{Competing interests}

The authors declare that they have no competing interests. 


\section{References}

1. Chadwick RB, Jiang GL, Bennington GA, Yuan B, Johnson CK, Stevens MW, Niemann TH, Peltomaki P, Huang $S$ and de la Chapelle A: Candidate tumor suppressor RIZ is frequently involved in colorectal carcinogenesis. Proc Natl Acad Sci USA 97: 2662-2667, 2000.

2. Jiang GL and Huang S: Adenovirus expressing RIZ1 in tumor suppressor gene therapy of microsatellite-unstable colorectal cancers. Cancer Res 61: 1796-1798, 2001.

3. Mandelbaum J, Bhagat G, Tang H, Mo T, Brahmachary M, Shen Q, Chadburn A, Rajewsky K, Tarakhovsky A, Pasqualucci L and Dalla-Favera R: BLIMP1 is a tumor suppressor gene frequently disrupted in activated B cell-like diffuse large B cell lymphoma. Cancer Cell 18: 568-579, 2010.

4. Wieser R: The oncogene and developmental regulator EVI1: Expression, biochemical properties, and biological functions. Gene 396: 346-357, 2007.

5. Shing DC, Trubia M, Marchesi F, Radaelli E, Belloni E, Tapinassi C, Scanziani E, Mecucci C, Crescenzi B, Lahortiga I, et al: Overexpression of SPRDM16 coupled with loss of p53 induces myeloid leukemias in mice. J Clin Invest 117: 3696-3707, 2007.

6. Deng Q and Huang S: PRDM5 is silenced in human cancers and has growth suppressive activities. Oncogene 23: 4903-4910, 2004.

7. Meani N, Pexximenti F, Deflorian G, Mione M and Alcalay M: The tumor suppressor PRDM5 regulates Wnt signaling at early stages of zebrafish development. PLoS One 4: e4273, 2009.

8. Burkitt Wright EMM, Spencer HL, Daly SB, Manson FDC, Zeef LAH, Urquhart J, Zoppi N, Bonshek R, Tosounidis I, et al: Mutations in PRDM5 in brittle cornea syndrome identify a pathway regulating extracellular matrix development and maintenance. Am J Hum Genet 88: 767-777, 2011.

9. Livak KJ and Schmittgen TD: Analysis of relative gene expression data using real-time quantitative PCR and the 2(-Delta Delta C(T)) method. Methods 25: 402-408, 2001.

10. Hohenauer T and Moore AW: The Prdm family: Expanding roles in stem cells and development. Development 139: 2267-2282, 2012.

11. Chang JC, Meredith DM, Mayer PR, Borromeo MD, Lai HC, $\mathrm{Ou}$ YH and Johnson JE: Prdm13 mediates the balance of inhibitory and excitatory neurons in somatosensory circuits. Dev Cell 25: 182-195, 2013.

12. Bäumer N, Tickenbrock L, Tschanter P, Lohmeyer L, Diederichs S, Bäumer S, Skryabin BV, Zhang F, Agrawal-Singh S, Köhler G, et al: Inhibitor of cyclin-dependent kinase (CDK) interacting with cyclin A1 (INCA1) regulates proliferation and is repressed by oncogenic signaling. J Biol Chem 286: 28210-28222, 2011.

13. Moncada-Pazos A, Obaya AJ, Fraga MF, Viloria CG, Capellá G, Gausachs M, Esteller M, López-Otín C and Cal S: The ADAMTS12 metalloprotease gene is epigenetically silenced in tumor cells and transcriptionally activated in the stroma during progression of colon cancer. J Cell Sci 122: 2906-2913, 2009.
14. El Hour M, Moncada-Pazos A, Blacher S, Masset A, Cal S, Berndt S, Detilleux J, Host L, Obaya AJ, Maillard C, et al: Higher sensitivity of Adamts12-deficient mice to tumor growth and angiogenesis. Oncogene 29: 3025-3032, 2010.

15. Wang D, Zhu T, Zhang FB and He C: Expression of ADAMTS12 in colorectal cancer-associated stroma prevents cancer development and is a good prognostic indicator of colorectal cancer. Dig Dis Sci 56: 3281-3287, 2011.

16. Zhang H, Zhu W, Su X, Wu S, Lin Y, Li J, Wang Y, Chen J, Zhou Y, Qiu P, et al: Triprolide inhibits proliferation and invasion of malignant glioma cells. J Neurooncol 109: 53-62, 2012.

17. Schmitz AA, Govek EE, Böttner B and Van Aelst L: Rho GTPases: Signaling, migration, and invasion. Exp Cell Res 261: $1-12,2000$

18. Wang J, Qian J, Hu Y, Kong X, Chen H, Shi Q, Jiang L, Wu C, Zou W, Chen Y, et al: ArhGAP30 promotes p53 acetylation and function in colorectal cancer. Nat Commun 5: 4735, 2014.

19. Yuan BZ, Miller MJ, Keck CL, Zimonjic DB, Thorgeirsson SS and Popescu NC: Cloning, characterization, and chromosomal localization of a gene frequently deleted in human liver cancer (DLC-1) homologous to rat RhoGAP. Cancer Res 58: 2196-2199, 1998.

20. Qian X1, Li G, Asmussen HK, Asnaghi L, Vass WC, Braverman R, Yamada KM, Popescu NC, Papageorge AG and Lowy DR: Oncogenic inhibition by a deleted in liver cancer gene requires cooperation between tensin binding and Rho-specific GTPase-activating protein activities. Proc Natl Acad Sci USA 104: 9012-9017, 2007.

21. Yuan BZ, Jefferson AM, Baldwin KT, Thorgeirsson SS, Popescu NC and Reynolds SH: DLC-1 operates as tumor suppressor gene in human non-small cell lung carcinomas. Oncogene 23: 1405-1411, 2004.

22. Karnoub AE, Symons M, Campbell SL and Der CJ: Molecular basis for Rho GTPase signaling specificity. Breast Cancer Res Treat 84: 61-71, 2004.

23. Ridley AJ: Rho proteins and cancer. Breast Cancer Res Treat 84: 13-19, 2004.

24. Gómez del Pulgar T, Benitah SA, Valerón PF, Espina C and Lacal JC: Rho GTPase expression in tumourigenesis: Evidence for a significant link. Bioessays 27: 602-613, 2005.

25. Verhaak RG, Hoadley KA, Purdom E, Wang V, Qi Y, Wilkerson MD, Miller CR, Ding L, Golub T, Mesirov JP, et al: Integrated genomic analysis identifies clinically relevant subtypes of glioblastoma characterized by abnormalities in PDGFRA, IDH1, EGFR, and NF1. Cancer Cell 17: 98-110, 2010.

This work is licensed under a Creative Commons Attribution-NonCommercial-NoDerivatives 4.0 International (CC BY-NC-ND 4.0) License. 